\title{
Model “Alloy” Specimens for MEMS-Based Closed-Cell Gas-Reactions
}

\author{
Lawrence F. Allard ${ }^{1}$, Harry M. Meyer III ${ }^{1}$, Dale K. Hensley ${ }^{2,}$ Wilbur C. Bigelow ${ }^{3}$ and Kinga A. Unocic ${ }^{1}$ \\ 1. Materials Science \& Technology Div., Oak Ridge National Laboratory, Oak Ridge, TN, USA. \\ 2. Center for Nanophase Materials Sciences, Oak Ridge National Laboratory, Oak Ridge, TN, USA. \\ ${ }^{3 .}$ Department of Materials Science \& Engineering, University of Michigan, Ann Arbor, MI, USA.
}

MEMS-based closed-cell gas-reaction specimen holders offer unique opportunities for in situ TEM/STEM studies of the behavior of samples such as catalyst powders at elevated temperatures and in gas environments up to a full atmosphere pressure [1,2]. Preparing a cell for catalyst (or nanoparticle) studies typically requires deposition onto the heater (or "E-chip) of the material either from the dry powder, or from a liquid suspension. Comparable studies of "bulk" materials, however, requires preparation of the bulk sample so that a thin slice of suitable geometry (e.g. electron transparent by a few microns in lateral extent) can be secured in some fashion to the E-chip membrane. This process can be conducted using focused-ion-beam (FIB)-milling procedures [3], with the caveat that the milling process typically leaves residual $\mathrm{Ga}$ on the slice surface, thereby complicating the reaction process. Nano-sized particles of alloy compositions are not readily available, and crushing alloy powders has also been problematical [4]. We describe here a potential new method for producing alloy specimens of controlled composition and geometry onto gas-cell heater membranes.

The basic idea for the model alloy specimens is to deposit "islands" of the desired alloy using a suitable vapor deposition technique where the elemental species deposit onto the E-chip membrane through a "mask" composed of an array of 1-2 $\mu \mathrm{m}$ diameter holes. The mask can be produced by FIB-milling techniques, using a SiN "window" device such as provided for the lower element of the closed-cell reactor holder. Alternatively, a commercially available Si chip with an array of holes in a SiN window membrane on the chip can be used (e.g. TEMwindows, a division of SiMPore Inc). This research demonstrates the preliminary results using this latter technique. Figure 1 shows an SEM image of the heater membrane of a Protochips Inc. E-chip; to align the Si grid chip with the SiN-covered "holes" in the E-chip, the Si grid chip is first carefully glued to a window device with the SiN window broken out, so that the evaporated species are isolated to a small area of the E-chip. Figure 2 shows a schematic of the cross section of the sandwich of these devices; the masking chips are aligned appropriately with the E-chip using a special precision-machined fixture. Our e-beam evaporator has sources aligned at a $24^{\circ}$ angle to the target, as shown in the figure. Figure 3 is an optical micrograph looking at the masked $\mathrm{Si}$ grid chip; the "stripes" are overlapped Al and Ni thin deposits caused by the (calculated) $114 \mu \mathrm{m}$ distance between the edges of the mask chip and the Si grid chip. Figure 4 shows an SEM image of the mask chip, with a corresponding SEM image of the island structure on the heater surface beneath the mask aligned and overlapped. The Ni stripe region is denoted by the yellow lines, and the Al by the orange lines. The compositions of the islands in the regions shown were determined by SEM-EDS. The precise composition of the co-deposited film was determined by XPS techniques, and was controlled to yield $50-50 \mathrm{Ni}-\mathrm{Al}$ by at\%. The image of the heater in Fig. 4 shows the area of Fig. 5 outlined; Fig. 5 is an optical micrograph of the two "holes" in the heater membrane on which islands $2.5 \mu \mathrm{m}$ in diameter were deposited. The rows of holes in this micrograph are labeled, with 2 rows of Al islands at the bottom, and 2 rows of $\mathrm{Ni}$ at the top. The 3-4 rows in the center of Fig. 5 are in the Ni-Al overlap region, with only $1 \mathrm{Ni}-\mathrm{Al}$ row extending over the bottom edge of the upper hole in Fig. 5 (i.e. "Hole Mid 2". This is confirmed by the STEM-EDS maps of hole Mid 2. Note also that the black spots in Fig. 5 and in Fig. 6 are $\mathrm{MgO}$ smoke particles, deposited as a marker. The size of the overlap regions of the $\mathrm{Ni}$ and $\mathrm{Al}$ 
deposits can be better controlled by mask geometry, but the individual islands will always suffer from at least a small misalignment, since the Si grid mask (or any other FIB-prepared mask) can never be placed directly against the heater surface. We are exploring the potential to use a commercial NiAl alloy target in an ion-sputter deposition system as a single-source target for depositing islands of uniform composition, for further in situ oxidation experiments. [5]

\section{References:}

[1] L.F. Allard, et al., Microsc. Microanal. 18, (2012) 656-666

[2] J.F. Creemer, et al., J. Microelectromechanical Systems, 19(2), (2010) 254-264

[3] M. Duchamp, Q. Xu, and R.E. Dunin-Borkowski, Microsc. Microanal., 20(6), (2014) 1638-1645

[4] K A Unocic et al., Oxid. Metals (2017) p.1.

[5] Research sponsored by the Laboratory Directed Research and Development program, ORNL, managed by UT-Battelle LLC for the US Department of Energy.
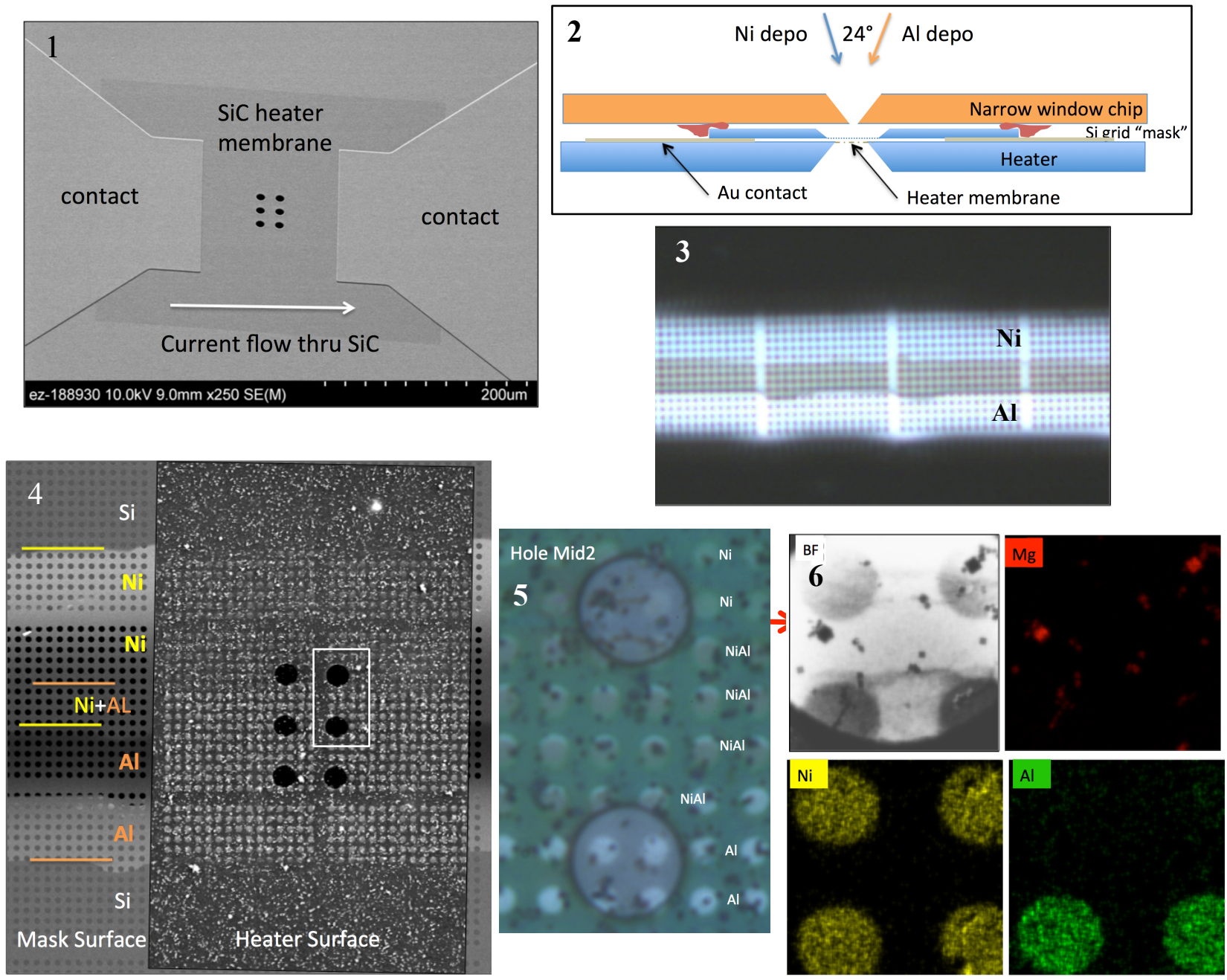

Figure 1. SEM image of Protochips E-chip heater membrane.

Figure 2. Schematic of structure of masked heater for deposition of NiAl islands.

Figure 3. Ni and Al "stripes" on the Si grid chip mask.

Figure 4. Overlap of SEM images of the mask chip and the heater surface; see text for details.

Figure 5. Optical micrograph of the heater chip area of the box in Fig. 4, with island compositions labeled.

Figure 6. BF image of hole "Mid 2" showing pure Ni and overlapped NiAl alloy islands. 